\title{
dilatance, dissipation d'énergie et critère de rupture tridimensionnel sous grandes déformations dans les matériaux granulaires
}

\author{
dilatancy, energy-dissipation \\ and tridimensional sailure criterion \\ under large strains in granular materials
}

\author{
E. FROSSARD \\ Ingénieur, Coyne \& Bellier*
}

\section{Résumé}

Cet article propose un schéma physique simple décrivant les aspects essentiels du comportement irréversible. A partir d'une analogie formelle entre le critère de Coulomb et les relations contraintes-dilatance de Rowe est mis à jour un principe énergétique élémentaire, sousjacent à ces équations, établies en symétrie axiale ou en déformation plane.

La traduction objective de ce principe mène alors à une équation de dissipation, tridimensionnelle. On détaille des résultats expérimentaux variés qui portent la trace de cette équation de dissipation.

On examine les conséquences théoriques de cette équation de dissipation sur le critère de rupture tridimensionnel sous grandes déformations, à l'état critique. La pyramide de Coulomb apparaît alors comme minimum de résistance et comme lieu des chemins de moindre énergie.

\section{Abstract}

A simple physical scheme is proposed in the paper, which describes essential features of irreversible behaviour. Proceeding from a formal analogy between Coulomb's criterion and Rowe's stress-dilatancy relations, an elemental energetic principle appears, underlying these equations, which are established for axisymetric conditions or place strain.

The objective expression of this principle leads to an energy-dissipation equation valid for tridimensional conditions. Various experimental results following the line of this energy-dissipation equation are detailed.

Theorical consequences of this energy-dissipation equation on critical state failure criterion are examined.

Coulomb's pyramid then appears both as a strengh minimum and as a locus of minimum energy paths. 


\section{INTRODUCTION}

Une certaine approche énergétique du comportement mécanique des sols a été développée, pour les matériaux fins, par les auteurs du modèle CAM-CLAY et de ses dérivés.

Pour les matériaux granulaires, et dans un autre ordre d'idées, ROWE (1962) et HORNE (1965) ont obtenu un résultat très convaincant à partir d'une modélisation microstructurale et d'un principe de minimisation d'énergie, en symétrie de révolution. L'usage d'un principe de minimisation d'énergie, parfois discuté, a conduit de JOSSELIN DE JONG (1976) à proposer une autre origine aux intrigantes équations de ROWE, fondée cette fois sur les seules lois de la friction, toujours dans le cas de la symétrie de révolution en contraintes.

L'objet de cet article est d'aller plus loin dans cette approche physique énergétique, sur les chemins tridimensionnels, et en la reliant à la notion de critère de rupture.

Le schéma original qui est présenté ici permet de décrire clairement nombre de caractères essentiels du comportement de ces matériaux. Comme il ne comporte qu'un seul paramètre intrinsèque, il est clair qu'il ne constitue pas un modèle complet.

Le développement des méthodes de calcul numérique en mécanique des sols a suscité la floraison de modèles de comportement, parfois sophistiqués, qui tentent d'en saisir l'intégralité.

Il semble à l'usage que certains calculs d'ouvrages réels pourraient souvent être aussi bien conduits avec des modèles simplifiés, plus proches de la caricature efficace que de la description minutieuse, dont la précision est souvent rendue superflue par lé incertitudes et inconnues qui peuvent subsister jusqu'à un stade avancé de la construction.

C'est dans cet esprit de recherche de schémas simples que s'inscrit cet article.

\section{UNE ANALOGIE FORMELLE ET SA TRADUCTION PHYSIQUE}

- Dans ce chapitre est détaillé le parallélisme qui relie le critère de rupture de Coulomb aux relations contraintes dilatance de Rowe, ainsi que la signification physique précise que l'on peut en tirer.

Le critère de Coulomb est une relation portant sur les seules contraintes, tandis que les relations de Rowe font intervenir à la fois les contraintes et vitesses de déformation ; aussi, afin d'établir ce parallélisme, considérera-t-on le critère de Coulomb lorsque le matériau atteint un régime stationnaire de déformation à volume constant, comme cela se produit après de larges déformations, à l'état critique.

- Dans les conditions du « triaxial * axisymétrique en compression, de la déformation plane, ou du « triaxial * axisymétrique en extension, la validité du critère de
Coulomb est largement reconnue, bien qu'il soit nécessaire de faire apparaître des angles de frottement internes distincts pour ces trois situations, dès que le matériau exhibe une certaine dilatance; cependant, on considère ici les conditions à volume constant de l'état critique.

Les relations de Rowe ont été établies à l'origine dans le cas de la symétrie de révolution, et plus tard étendues au cas de la déformation plane sur une base expérimentale (Rowe (1969)). Ces relations sont en principe valides pour toute déformation à volume variable.

\subsection{Les cas de symétrie axiale et de déformation plane}

- Considérons d'abord le cas du «triaxial » compression. Les contraintes de compression et déformations en contraction sont comptées positivement, la coaxialité des tenseurs $\underline{\sigma}$ et $\underline{\varepsilon}$ est supposée réalisée, on se place dans le repère formé par les directions principales, numérotées dans l'ordre décroissant des contraintes principales $\sigma_{1}>\sigma_{2}>\sigma_{3}$. Les vitesses de déformation principale, ainsi numérotées, n'ont donc pas d'ordre défini.

- Les matériaux granulaires ne résistant guère à la traction, on suppose que les contraintes principales sont toutes des compressions.

- Le critère de Coulomb

$$
\left(\sigma_{1}-\sigma_{3}\right)=\sin \phi
$$

s'écrit aussi :

$$
\sigma_{1} / \sigma_{3}=\operatorname{tg}^{2}(\pi / 4+\phi / 2)
$$

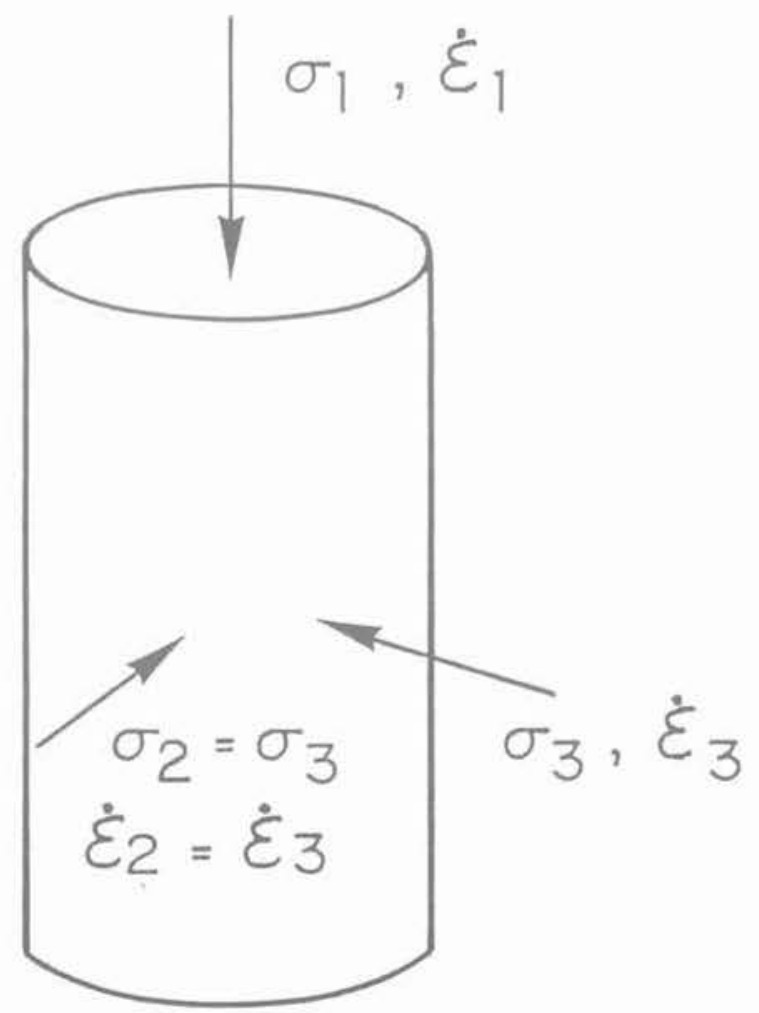

Essai triaxial en compression. 
Dans les conditions de volume constant, cette dernière expression peut être transformée, en tenant compte de la condition $\dot{\varepsilon}_{v}=\hat{\varepsilon}_{1}+2 \dot{\varepsilon}_{3}=0$, qui s'écrit aussi $\dot{\varepsilon}_{1} / 2 \dot{\varepsilon}_{3}=-1$

On obtient ainsi par multiplication :

$\sigma_{1} \dot{\varepsilon}_{1} / 2 \sigma_{3} \dot{\varepsilon}_{3}=-\operatorname{tg}^{2}\left(\pi / 4+\phi_{c} / 2\right)$ où $\phi_{\mathrm{cv}}$ est l'angle de frottement interne à volume constant de l'état critique.

- La relation de Rowe s'écrit ici :

$$
\sigma_{1} \dot{\varepsilon}_{3} / 2 \sigma_{3} \dot{\varepsilon}_{3}=-\operatorname{tg}^{2}(\pi / 4+\phi / 2)
$$

où $\phi$, est une constante expérimentale, intermédiaire entre l'angle de friction physique grain sur grain $\phi_{\mu}$ et l'angle $\phi_{\mathrm{c} v}$. Les valeurs mesurées pour $\phi_{1}$ sont voisines de $\phi_{u}$ dans le cas du triaxial compression sur matériau dense.

- Les situations de la déformation plane et du * triaxial extension » s'étudient sans peine de façon similaire. En tenant compte des particularités de ces diverses situations, on obtient le tableau suivant:
Le parallélisme est frappant, non seulement entre les colonnes du tableau I, mais aussi entre les lignes. Physiquement, ces expressions indiquent que le rapport de certaines puissances développées par les contraintes est constant.

- Examinons d'abord les constantes:

- d'après les résultats de CORNFORTH (1964), repris par LAMBE et WITHMAN (1969), les frottements internes à volume constant seraient très voisins pour les trois chemins, à l'état critique.

$$
\phi_{\mathrm{cv} 1} \cong \phi_{\mathrm{c} 2} \cong \phi_{\mathrm{cv} 3}
$$

- d'après les résultats de HORNE (1969) et ceux de l'auteur (1983), l'angle de frottement interne à volume constant $\phi_{\alpha 1}$ au triaxial compression excède l'angle de friction physique $\phi_{4}$ de quelques degrés. Or $\phi_{1}$ est compris entre $\phi_{c v}$ et $\phi_{\mu}$. La distinction entre tous ces angles suppose une précision qui peut paraître un peu superflue dans l'optique de certains calculs d'ouvrages. L'auteur propose donc de remplacer tous ces angles par une seule constante, notée $\psi$ et appelée « angle d'écoulement *.

$$
0<\psi<\pi / 2
$$

\begin{tabular}{|c|c|c|}
\hline $\begin{array}{l}\text { Chemin de } \\
\text { sollicitation }\end{array}$ & $\begin{array}{l}\text { Critère de Coulomb } \\
\text { rupture à volume constant }\end{array}$ & $\begin{array}{l}\text { Relations de Rowe } \\
\text { déformation à volume variable }\end{array}$ \\
\hline $\begin{array}{l}\text { 1. Axisymétrique } \\
\text { en compression } \\
\sigma_{1} \text { axial } \\
\sigma_{2}=\sigma_{3} \text { radial }\end{array}$ & $\sigma_{1} \dot{\varepsilon}_{1} / 2 \sigma_{3} \dot{\varepsilon}_{3}=-\operatorname{tg}^{2}\left(\pi / 4+\phi_{\text {cvv }} / 2\right)$ & $\sigma_{1} \hat{\varepsilon}_{1} / 2 \sigma_{3} \hat{\varepsilon}_{3}=-\operatorname{tg}^{2}\left(\pi / 4+\phi_{n 1} / 2\right)$ \\
\hline $\begin{array}{l}\text { 2. Déformation plane } \\
\dot{\varepsilon}_{2}=0\end{array}$ & $\sigma_{1} \dot{\varepsilon}_{1} / \sigma_{3} \dot{\varepsilon}_{3}=-\operatorname{tg}^{2}\left(\pi / 4+\phi_{\mathrm{ov} 2} / 2\right)$ & $\sigma, \dot{\varepsilon}_{1} / \sigma_{3} \dot{\varepsilon}_{3}=-\operatorname{tg}^{2}\left(\pi / 4+\phi_{12} / 2\right)$ \\
\hline $\begin{array}{l}\text { 3. Axisymétrique } \\
\text { en extension } \\
\sigma_{1}=\sigma_{2} \text { radial } \\
\sigma_{3} \text { axial }\end{array}$ & $2 \sigma, \dot{\varepsilon}_{1} / \sigma_{3} \dot{\varepsilon}_{3}=-\operatorname{tg}^{2}\left(\pi / 4+\phi_{c o d} / 2\right)$ & $2 \sigma_{1} \dot{\varepsilon}_{1} / \sigma_{3} \dot{\varepsilon}_{3}=-\operatorname{tg}^{2}\left(\pi / 4+\phi_{t 3} / 2\right)$ \\
\hline
\end{tabular}

Tableau I. - L'analogie formelle entre le critère de Coulomb à l'état critique et les relations contraintesdilatance de Rowe

- Le rapport de puissances qui apparait au premier membre des expressions du tableau 1 exhibe:

- au numérateur, la puissance développée dans les directions principales pour lesquelles la vitesse de déformation est une contraction, c'est-à-dire la puissance développée dans les directions principales par lesquelles le matériau reçoit de l'énergie du système de sollicitation, que l'on propose de nommer «puissance reçue », notée $\mathrm{P}_{+}$;

- au dénominateur, la puissance développée dans les directions principales par lesquelles le matériau rend de l'énergie au milieu extérieur (vitesses de déformation en extension), que l'on propose de nommer «puissance retransmise „ notée $\mathrm{P}$.

On note de plus que la somme algébrique des puissances $\mathrm{P}_{+}$et $\mathrm{P}_{-}$, dans les expressions du tableau I, est égale à la puissance des efforts intérieurs:

$$
P_{+}+P_{-}=\sum_{1} \sigma_{t} \dot{\varepsilon}_{i}
$$

- A partir de ces définitions, l'unité formelle du tableau I s'exprime simplement: les puissances recues $\mathrm{P}_{+}$et et retransmises $\mathrm{P}_{-}$sont en rapport constant, qui s'écrit :

$$
P_{+} / P=-\operatorname{tg}^{2}(\pi / 4+\psi / 2)
$$

On sait relier ce qui précède aux caractères physiques êlémentaires de la microstructure du matériau et des actions mutuelles entre ses éléments, c'est-à-dire des contacts à frottement sec, dans le cas d'une sollicitation axisymétrique (HORNE, 1965; DE JOSSELIN DE JONG, 1976) ou dans le cas d'un matériau bidimensionnel (CAMBOU, 1985). Cependant, dans le cas plus général d'une sollicitation tridimensionnelle, on ne sait guère pour linstant relier ce qui précède à des causes physiques élémentaires.

Aussi l'auteur propose de poser comme principe la constance du rapport entre $\mathrm{P}_{+}$et $\mathrm{P}_{-}$. 


\subsection{La transposition tridimensionnelle}

Tout ce qui précède a trait à des chemins de sollicitation bien particuliers, bidimensionnels en fait. Pour étendre le principe précédent aux chemins tridimensionnels, il convient d'abord de trouver une définition objective des puissances $\mathrm{P}_{+}$et $\mathrm{P}_{-}$, qui est donnée par les formulations suivantes:

$$
\begin{aligned}
& P_{+}=\left(\sum_{i} \sigma_{i} \varepsilon_{i}+\sum_{i}\left|\sigma_{i} \varepsilon_{i}\right|\right) / 2 \\
& P_{-}=\left(\sum_{i} \sigma_{i} \varepsilon_{i}-\sum_{i}\left|\sigma_{i} \varepsilon_{i}\right|\right) / 2
\end{aligned}
$$

Ces définitions vérifient bien sûr la condition:

$$
\mathrm{P}_{+}+\mathrm{P}_{-}=\sum \sigma_{i} \dot{\varepsilon}_{i}
$$

La formulation tridimensionnelle du principe physique proposé est donc:

$$
\begin{aligned}
& \quad P_{+} / P_{-}=\left(\sum_{1} q \varepsilon+\sum_{1}|q \varepsilon|\right) /\left(\sum_{1} q \varepsilon-\sum_{1}|q \varepsilon|\right) \\
& =-\operatorname{tg}^{2}(\pi / 4+\psi / 2)
\end{aligned}
$$

Ceci s'écrit encore, de façon plus simple:

$$
\sum \sigma_{i} \dot{\varepsilon}_{i}=\sin \psi \cdot \sum\left|\sigma_{i} \dot{\varepsilon}_{i}\right|
$$

- Cette équation, déjà établie par lauteur par une autre voie (1983), écrit l'égalité de la puissance des efforts intérieurs, au premier membre, avec un second membre toujours positif. Elle prévoit donc que le matériau absorbe toujours de l'énergie dès qu'il y a déformation $(\varepsilon \neq 0)$ sous contraintes $(\underline{\sigma} \neq \underline{0})$, ce qui est concevable si cette énergie est dissipée au fur et à mesure. Bien que le terme «flow rule» ou "loi d'écoulement ", défini par certains mécaniciens des sols, s'applique à la nature de l'équation (3), l'auteur propose de la nommer plutôt équation de dissipation, terme qui correspond mieux à sa nature physique.

- Par le jeu des valeurs absolues au deuxième membre, l'équation de dissipation change de forme lorsque changent de signe une ou plusieurs vitesses de déformation principale. (Les contraintes principales effectives sont toujours en compression dans les matériaux granulaires, elles ne changent pas de signe). On voit donc s'introduire ici, à partir d'une hypothèse physique sur les flux d'énergie, la notion de zone tensorielle développée depuis longtemps par F. DARVE (1983).

- Diverses conséquences de l'équation (3) ont déjà été détaillées en 1983, elle permet bien sûr de retrouver les relations de Rowe ainsi que le critère de Coulomb à volume constant, en symétrie axiale ou en déformation plane; une écriture au moyen d'invariants tensoriels en a été donnée à cette occasion.

\section{LA TRACE EXPÉRIMENTALE}

Les résultats qui fondent l'analyse théorique du chapitre précédent ont été acquis par de nombreuses expériences; on en propose ici une illustration, à partir d'éléments en majorité publiés dans la littérature, et reproduits sous leur forme originale. Ces résultats ont tous trait aux conditions drainées.

\subsection{En symétrie axiale, sous sollicitation monotone}

- La relation de Rowe pour l'essai triaxial en compression s'écrit aussi :

$$
\sigma_{1} / \sigma_{3}=\left(1-\dot{\varepsilon}_{v} / \dot{\varepsilon}_{1}\right) \cdot \operatorname{tg}^{2}(\pi / 4+\psi / 2)
$$

Cette expression prévoit que, dans le diagramme contraintes-dilatance, c'est-à-dire le plan de coordonnées :

$\left(x=1-\dot{\varepsilon}_{v} / \dot{\varepsilon}_{1} ; y=\sigma_{1} / \sigma_{3}\right)$, la trace de l'essai est une droite $(y=k x)$, indépendante de l'état initial. La figure $1^{* *}$, qui décrit des essais sur un sable calcaire concassé pour trois densités initiales différentes, sous un même confinement $\sigma_{3}=0.05 \mathrm{MPa}$, traduit cette propriété: les traces des essais dans le diagramme contraintes-dilatance (fig. 1b) sont effectivement rassemblées sur une même droite.

- Si la densité initiale est maintenue constante, mais que la contrainte de confinement varie d'un essai à lautre, on sait que l'effet de pic s'affaiblit lorsque le confinement augmente, à cause de l'affaiblissement de la dilatance.

Notons $\phi_{\text {pic }}$ l'angle de frottement interne au pic, défini par $\sin \phi_{\text {pic }}=\left[\left(\sigma_{1}-\sigma_{3}\right) /\left(\sigma_{1}+\sigma_{3}\right)\right]_{\text {plc }}$ La relation (4) relie $\phi_{\text {pic }}$ au taux de dilatance observé au pic, par:

$$
\operatorname{tg}^{2}\left(\pi / 4+\phi_{\text {Dic }} / 2\right)=\left(1-\dot{\varepsilon}_{v} / \dot{\varepsilon}_{1}\right)_{p i c} \cdot \operatorname{tg}^{2}(\pi / 4+\psi / 2)
$$

La figure $2^{* *}$ montre des résultats au pic obtenus par CHARLES et WATTS (1980) par des essais triaxiaux drainés $\varnothing 230 \mathrm{~mm}$ sur un gravier de basalte concassé, pour des contraintes de confinement comprises entre $0,027 \mathrm{MPa}$ et $0,7 \mathrm{MPa}$. La courbe théorique tracée par l'auteur pour $\psi=45^{\circ}$ représente assez bien les points expérimentaux, ce qui traduit l'indépendance approximative de $\psi$ par rapport à la pression de confinement.

On trouvera dans Rowe (1969), de nombreuses rếérences expérimentales plus détaillées.

Les résultats de BARDEN et KHAYATT (1966) indiquent que les relations contraintes-dilatance de Rowe restent valides au cours de sollicitations cycliques en symétrie axiale.

\footnotetext{
** reproduites avec l'autorisation de la revue Géotechnique.
} 


\section{Essai triaxial en compression}

\section{Fig. 1 - A Confinement \\ constant et porosité initiale variable.}

(a) Courbes d'essai

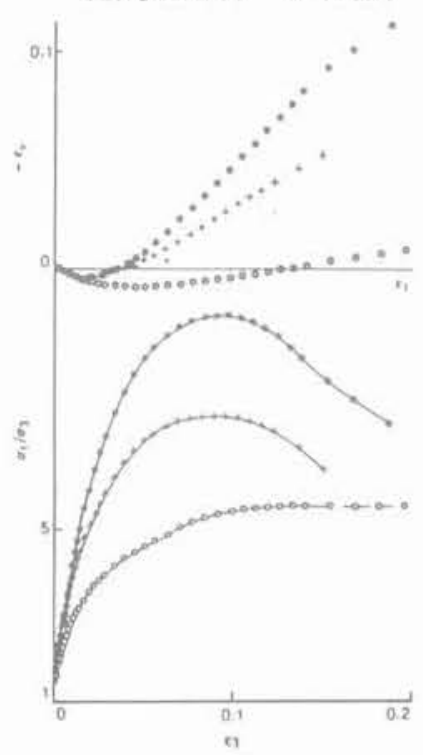

(b) Diagramme

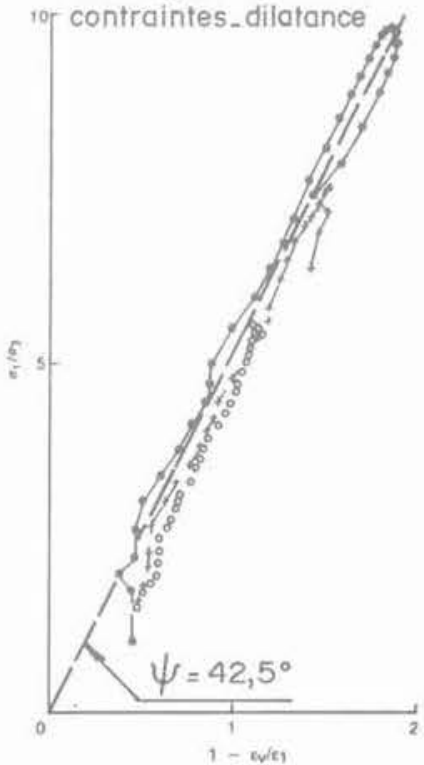

Fig. 2 - A porosite

initiale constante

et confinement variable.

Résultats au pic.

\subsection{En déformation plane}

HUGUES, WROTH et WINDLE (1977) rapportent une série d'essais réalisés par STROUD à l'Université de Cambridge, avec des densités et confinements variés, sur un appareil de déformation plane avec rotation d'axes (cisaillement simple).

Les courbes d'essai sont reproduites figures $3^{\prime}$ et $4^{\text {**** }}$, où l'on note que la distorsion cumulée $\sum \dot{\gamma}$ a été

poussée jusqu'à $50 \%$ dans certains essais.

Les figures $3 \mathrm{~b}$ et $4 \mathrm{~b}$ reproduisent la trace de ces essais dans le plan de coordonnées:

$$
\left\{\begin{array}{l}
\mathrm{x}=\left(\sigma_{1}-\sigma_{3}\right) /\left(\sigma_{1}+\sigma_{3}\right)=\mathrm{t}^{\prime} / \mathrm{s}^{\prime} \\
\mathrm{y}=-\left(\varepsilon_{1}+\varepsilon_{3}\right) /\left(\dot{\varepsilon}_{1}-\xi_{9}\right)=\dot{v} / \dot{\gamma}
\end{array}\right.
$$

On peut montrer sans peine à partir de l'équation (3) que la trace des essais de déformation plane dans ce plan est théoriquement une hyperbole d'équation:

$$
y=(x-\sin j \psi) /(x \sin \psi-1)
$$

La coincidence avec la courbe théorique tracée par l'auteur pour $\psi=36^{\circ}$ est nette sur les figures $3 \mathrm{~b}$ et $4 b$.

Un écart entre la prévision théorique et la tendance expérimentale apparaît sur la figure $4 \mathrm{~b}$, au tout début des essais (zone OA approximativement). Cette disposition traduit vraisemblablement l'effet d'un petit domaine élastique, il en est de même au tout début des essais triaxiaux de la figure 1.
BARDEN, ISMAIL et TONG (1969) détaillent des résultats d'essais sur un sable roulé réalisés à l'Université de Manchester avec un appareil de déformation plane sans rotation d'axes, quí indique la validité des relations de dilatance sur une large plage de pression de confinement (de 0,14 à $6 \mathrm{MPa}$ ).

\subsection{En chemin tridimensionnel cyclique}

- Les figures 5 et 6 reproduisent des essais effectués sur un sable dense à la presse tridimensionnelle de l'Institut de Mécanique de Grenoble et communiqués à l'auteur par J. LANIER en 1984.

Pour tester la validité de la relation de Rowe sur chemin tridimensionnel, LANIER utilise le plan dont les coordonnées sont:

- en abscisses, l'énergie reçue depuis le début de l'essai :

$$
X(T)=\int_{0}^{T} P+(t) d t
$$

- en ordonnées, le travail des efforts intérieurs ou énergie totale de déformation, cumulé depuis le début de l'essai :

$$
Y(T)=\int_{0}^{T}\left[P_{+}(t)+P_{-}(t)\right] d t
$$

L'équation de dissipation (3) prévoit que: $\left[P_{+}(t)+P_{-}(t)\right] / P_{+}(t)$ 


\section{Essai en déformation plane}

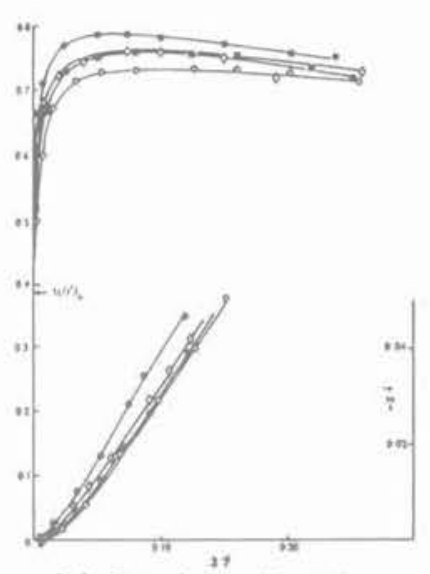

(a) Courbes d'essai

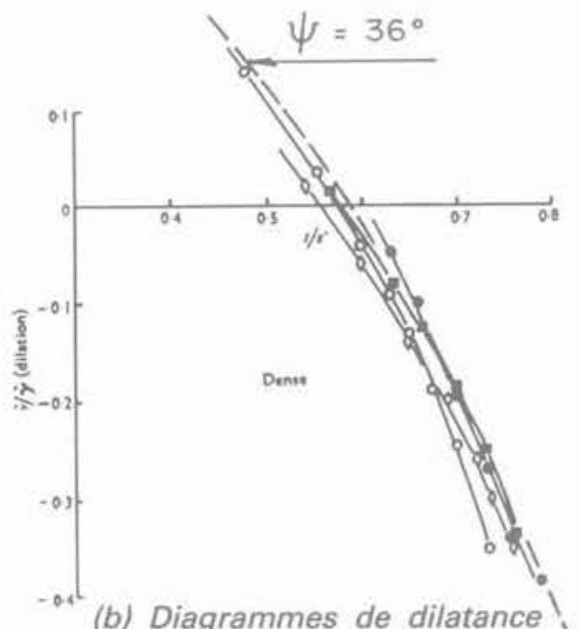

Fig. 3 - Matériau dense.

La trace de l'essai dans le plan $[\mathrm{X}(\mathrm{T}), \mathrm{Y}(\mathrm{T})]$, que l'on propose de nommer ici diagramme d'énergie, est donc théoriquement une droite, ce qui est étonnamment bien vérifié sur les figures $5 \mathrm{~b}$ et $6 \mathrm{~b}$.

L'usage des coordonnées intégrales $[X(T), Y(T)]$ a bien entendu pour effet de lisser les fluctuations instantanées, mais par ailleurs une éventuelle dérive systématique par rapport à la linéarité théorique prévue par (3) n'en serait que mieux soulignée.

- La figure 5 décrit un chemin axisymétrique en contrainte qui consiste en une sollicitation équivalente à un essai triaxial en compression suivi d'un décharge - avec passage aux conditions du triaxial extension puis d'une recharge - avec retour aux conditions du triaxial compression.

La droite donnée par la trace de l'essai dans le diagramme d'énergie, figure $5 \mathrm{~b}$, présente une pente valant environ 0,66 , ce qui correspond à un angle d'écoulement $\psi=29,5^{\circ}$.

On note enfin sur la figure $5 \mathrm{~b}$ que si le passage vers l'extension (repère (1) sur la figure 5) est marqué par un léger décrochement en $y$, dans lequel on peut

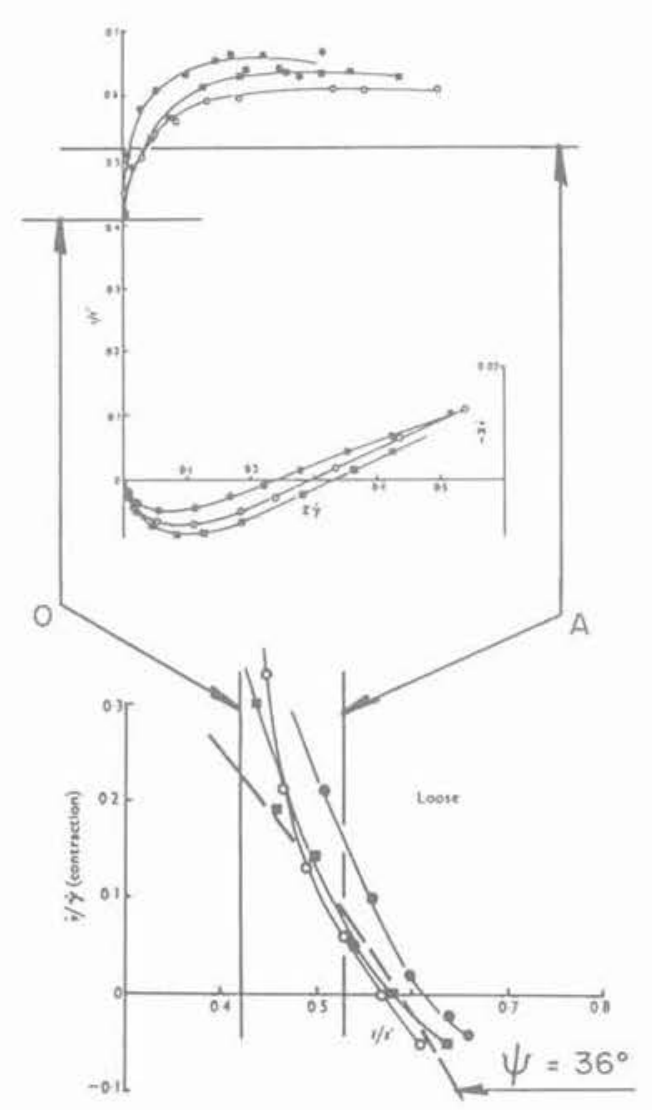

Fig. 4 - Matériau lâche.

voir la restitution rapide d'une partie de l'énergie stockée élastiquement dans l'éprouvette, le retour vers la compression (repère (2)) est pratiquement indécelable,

- La figure 6 décrit un chemin tridimensionnel dans lequel sont maintenus constants

$$
\left\{\begin{array}{l}
{\left[\sigma_{x}+\sigma_{y}+\sigma_{z}=1,5 \mathrm{MPa}\right.} \\
\left(\sigma_{z}-\sigma_{y}\right) /\left(\alpha_{x}-\sigma_{y}\right)=0,5
\end{array}\right.
$$

La droite donnée par la trace de l'essai dans le diagramme d'énergie de déformation, figure $6 \mathrm{~b}$, présente une pente d'environ 0,68 , qui correspond à un angle d'écoulement $\psi=31^{\circ}$.

- La figure 7 représente les chemins de ces essais dans l'espace des contraintes principales ordonnées $\sigma_{1}>\sigma_{2}>\sigma_{3}$.

On constate que ces chemins constituent un échantillonnage réduit mais régulier des directions possibles dans le secteur $\sigma_{1}>\sigma_{2}>\sigma_{3}$.

La variation de $1,5^{\circ}$ trouvée dans l'angle d'écoulement $\psi$ sur ces chemins n'apparaît pas à l'auteur comme très significative. 
(a) Courbes d'essai

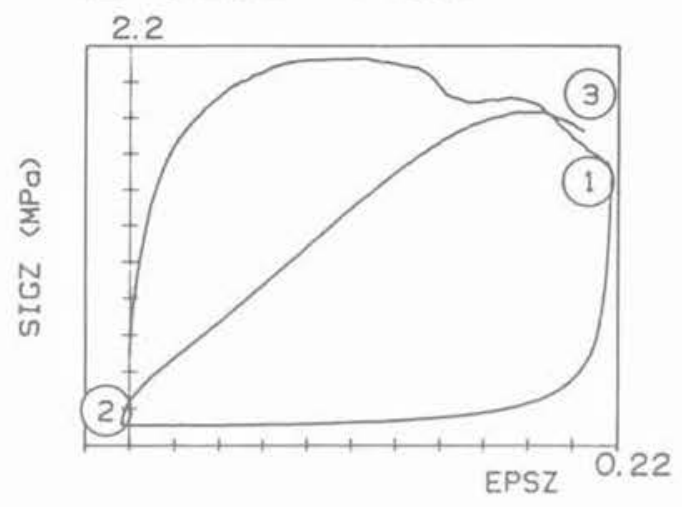

(b) Diagramme d'énergie
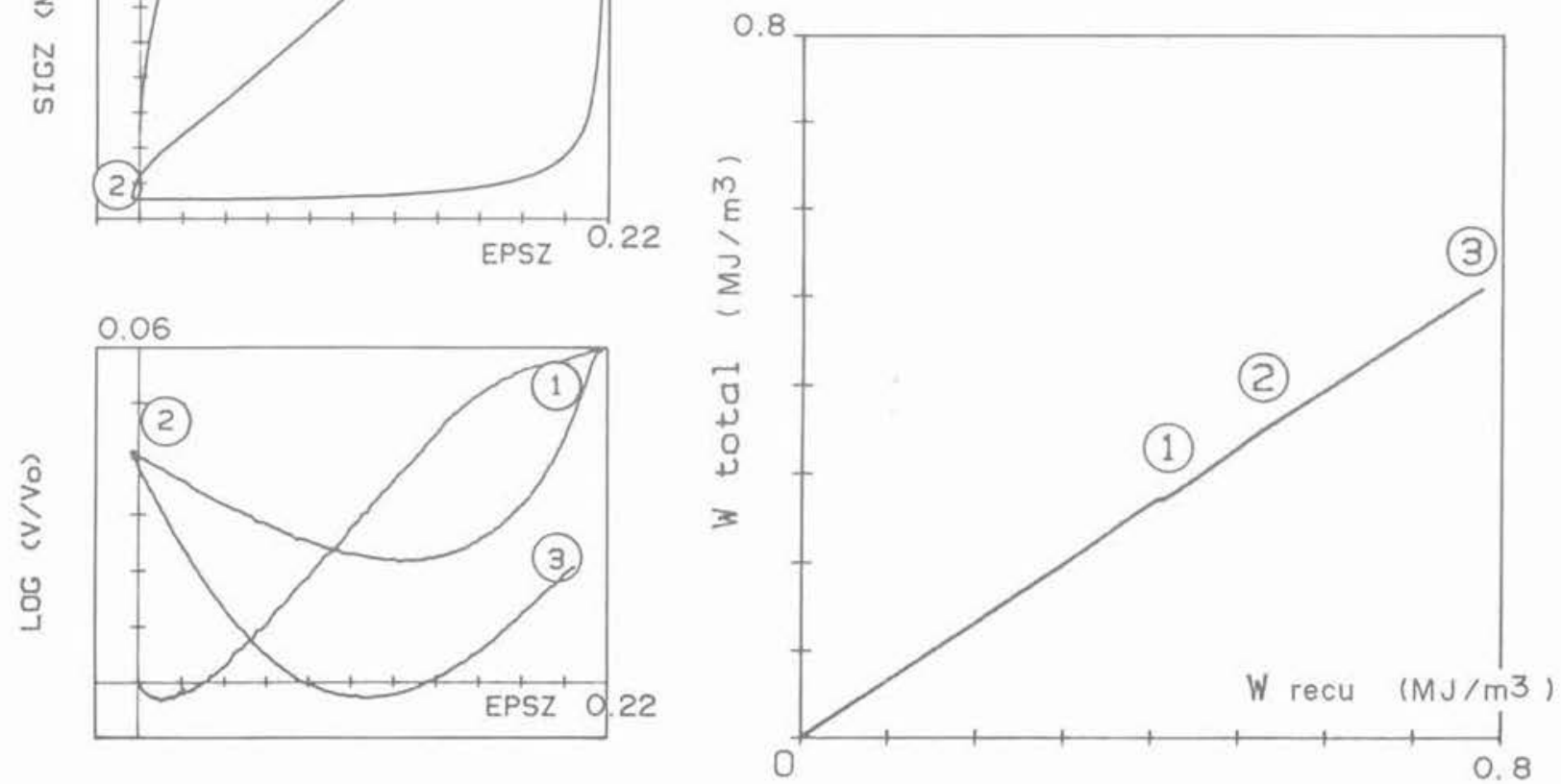

Fig. 5. - Essai tridimensionnel cyclique (J. Lanier, 1984) $\sigma_{x}=\sigma_{y}=0.5 \mathrm{MPa}$.

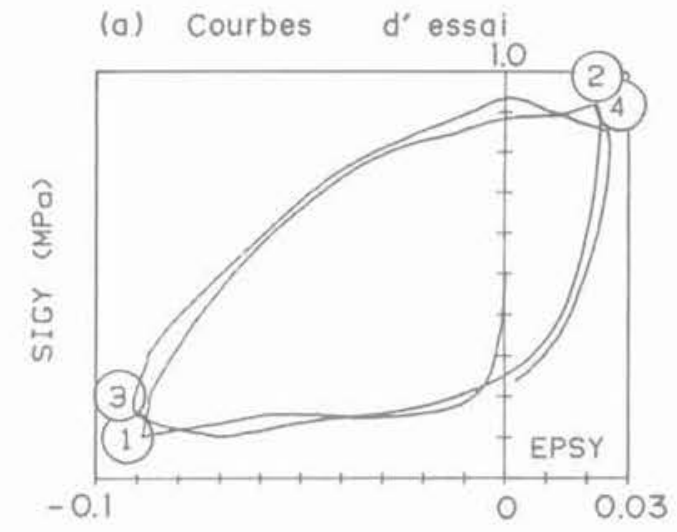

(b) Diagramme d'énergie
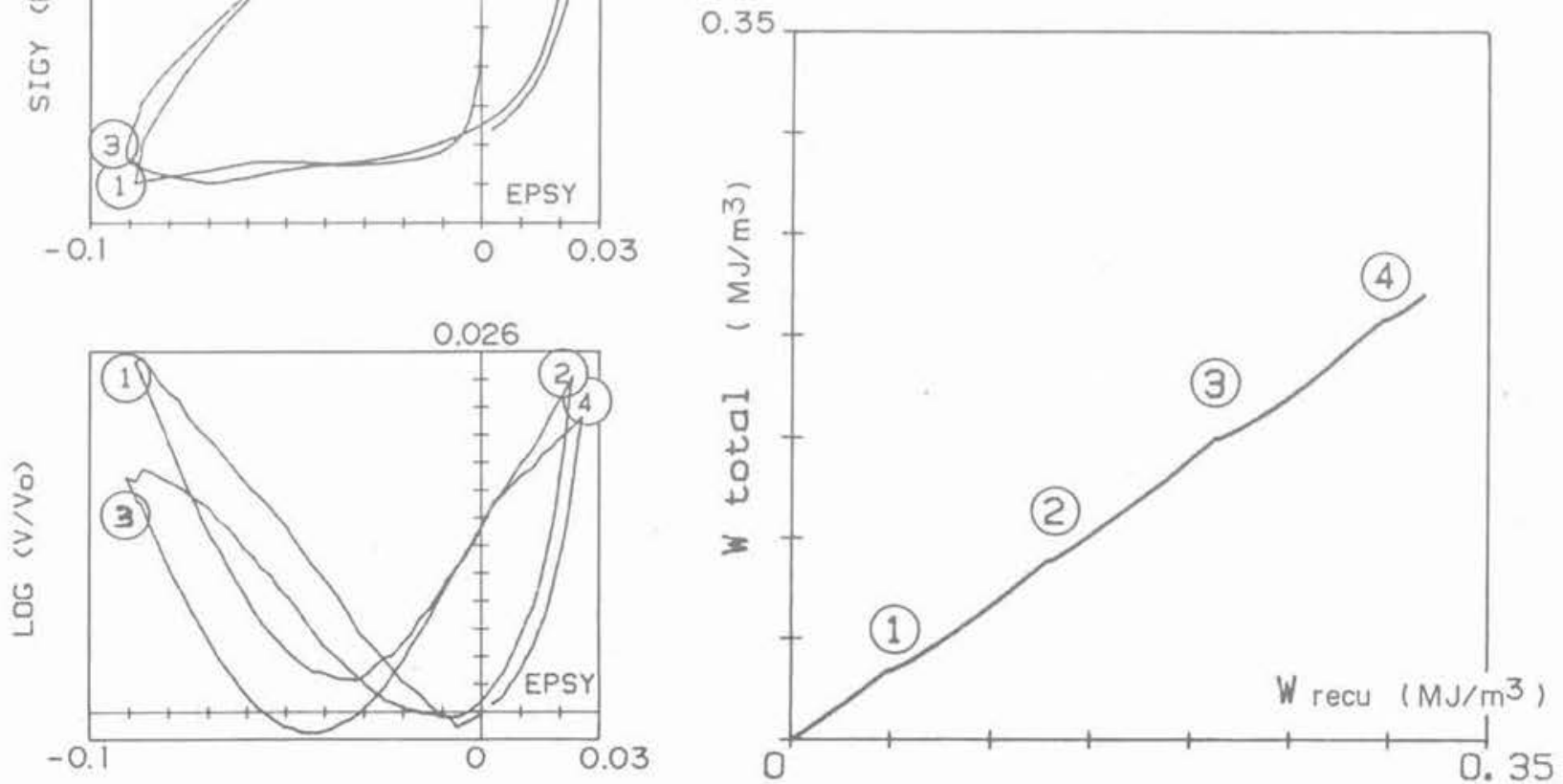

Fig. 6. - Essai tridimensionnel cyclique (J. Lanier, 1984) $\mathrm{Pm}=0.5 \mathrm{MPa} ; b=0.5$. 
(a) dans l'espace fixe des directions principales lié au laboratoire (b) dans l'espace des contraintes principales ordonnées

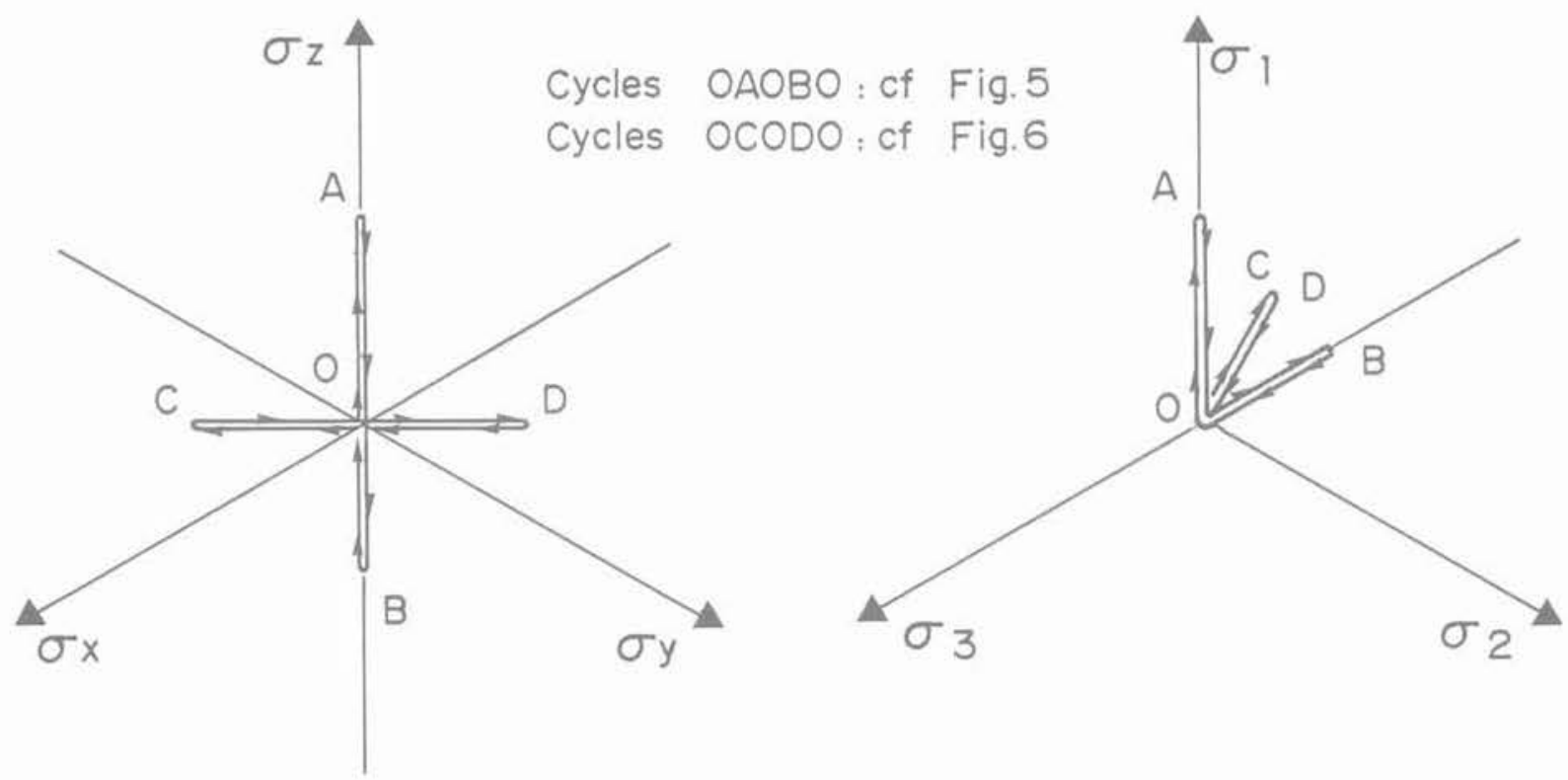

Fig. 7. - Représentation des cycles des figures 5 et 6 .

\section{LE CRITÈRE \\ DE RUPTURE TRIDIMENSIONNEL SOUS GRANDES DÉFORMATIONS}

L'équation de dissipation (3), bien que reposant sur des résultats acquis sur des chemins bidimensionnels, permet une description de situations tridimensionnelles, qui semble confirmée par l'expérience. Elle donne comme critère de rupture à volume constant celui de Coulomb, sur ces chemins simples que sont la symétrie axiale et la déformation plane. On analyse maintenant ce qui se passe en dehors de ces chemins simples.

\subsection{Le cadre physique et analytique, la relation déviatoire}

- Le critère de rupture sous grandes déformations est la trace, dans l'espace des contraintes, du régime d'écoulement limite qui peut être observé après de larges déformations monotones, lorsque le volume spécifique devient stationnaire. Ce régime limite, connu depuis longtemps (TAYLOR, 1948) est l'origine de la notion d'état critique (SCHOFIELD et WROTH, 1968).

- La condition de volume constant $\dot{\varepsilon}_{\nu}=0=\dot{\varepsilon}_{1}$ $+\dot{\varepsilon}_{2}+\dot{\varepsilon}_{3}$, et l'équation de dissipation (3), ne suffisent pas pour éliminer les vitesses de déformation et en tirer la relation sur les seules contraintes qui définit ce critère de rupture, il manque une relation scalaire entre $\sigma$ et $\dot{\varepsilon}$.

- Il existe un lien, observé par l'expérience, entre les déviateurs de contrainte et de vitesses de déformation, que F. DARVE (1983) dénomme loi d'écoulement dérivatoire. Usuellement ce lien est représenté comme une correspondance scalaire entre les invariants définissant les positions angulaires des déviateurs dans le repère principal.

Il peut également être représenté comme une relation liant les paramètres $\mathrm{b}=\left(\sigma_{2}-\sigma_{3} /\left(\sigma_{1}-\sigma_{3}\right)\right.$ et son homologue $\mathrm{C}=\left(\dot{\varepsilon}_{2}-\dot{\varepsilon}_{3}\right) /\left(\dot{\varepsilon}_{1}-\dot{\varepsilon}_{3}\right)$, que lon nommera ici e relation déviatoire $*$.

- Dans ce qui suit, on admet a priori qu'à l'état critique cette relation déviatoire $b=f(c)$ est continue, monotone croissante, et donc inversible; comme, par convention $0<\mathrm{b}<1$, on admet par symétrie que $0<\mathrm{c}<1$. Dans un premier temps, on s'attache à la forme du critère de rupture, à relation déviatoire donnée: dans un deuxième temps on relie cette notion de relation déviatoire aux chemins de moindre énergie.

\subsection{Détermination du critère de rupture pour une relation déviatoire donnée}

- Le critère de rupture est donné par la résolution du système formé par:

- l'équation de dissipation

- la condition de volume constant

- la relation déviatoire

(5)

$$
\begin{aligned}
& \sum \sigma_{1} \dot{\varepsilon}_{i}^{\prime}=\sin \psi \sum\left|\sigma_{i} \dot{\varepsilon}_{i}\right| \\
& \sum \dot{\varepsilon}_{i}=0 \\
& \left(\sigma_{2}-\sigma_{3}\right) /\left(\sigma_{1}-\sigma_{3}\right)=\mathrm{f}\left[\left(\varepsilon_{2}-\varepsilon_{3}\right) /\left(\varepsilon_{1}-\varepsilon_{3}\right)\right]
\end{aligned}
$$


- La présence de valeurs absolues dans l'équation de dissipation incite à examiner séparément tous les cas possibles, qui heureusement se réduisent à 2 (Cf Annexe 1), correspondant à deux modes de déformations tridimensionnels fondamentaux, séparés par un mode frontière en déformation plane

- le mode de déformation nommé model qui recouvre les régimes de déformation intermédiaires entre l'essai triaxial en compression et la déformation plane, pour lesquels, à volume constant, $0<\mathrm{c}<1 / 2$. soit :

$$
\left\{\begin{array}{l}
\dot{\varepsilon}_{1}>0 \\
\dot{\varepsilon}_{2}<0 \\
\dot{\varepsilon}_{3}<0
\end{array}\right.
$$
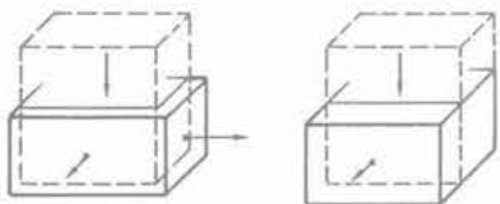

MODE I
DEFORMATION PLANE

Essai en déformation plane.

- Le mode Il qui recouvre les régimes de déformation intermédiaires entre la déformation plane et le triaxial extension, pour lesquels, à volume constant $\frac{1}{2}<c<1$, soit :

$$
\left\{\begin{array}{l}
\dot{\varepsilon}_{1}>0 \\
\dot{\varepsilon}_{2}>0 \\
\dot{\varepsilon}_{3}<0
\end{array}\right.
$$

- Pour chacun des modes, on peut alors résoudre analytiquement le système (5), suivant la méthode exposée en annexe II. Comme on la vu au chapitre 1. le critère de rupture obtenu «touches la pyramide de Coulomb, en symétrie axiale en contraintes $(b=0$ ou $b=1)$ ou en déformation plane ( $c=$ $1 / 2$ ). On montre en annexe II que, pour une relation déviatoire donnée $b=f(c)$, le critère de rupture correspondant est toujours à l'extérieur de la pyramide de Coulomb.

Les figures 8,9 et 10 présentent trois exemples:

- Figure 8, la relation déviatoire est $b=c$, les déviateurs en contraintes et vitesses de déformation sont colinéaires. Les domaines correspondant aux modes I et II couvrent des secteurs angulaires égaux, dans chacun d'eux le critère est convexe.

- Figure 9, la relation déviatoire est $b=c^{\alpha}, \alpha$ étant tel que, en déformation plane, on ait $\sigma_{2}=\sqrt{\sigma_{1} \sigma_{3}}$ (soit ici : $\mathrm{b}(1 / 2)=(1-\operatorname{tg} \psi / 2) / 2)$ suivant un résultat de GREEN cité par RAMAMURTHY et TOKHI (1981).
- Figure 10, la relation déviatoire, définie par $[c=1 / 2)$, sinon $b=0$ et $c<1 / 2$, ou bien $b=1$ et $c>1 / 2]$ est un cas limite de fonction $f$ envisagée en 3.1 .

Le critère obtenu est précisément la pyramide de Coulomb. Par rapport aux exemples précédents, le domaine de la déformation plane s'étend au détriment des modes I et II, réduits à des droites.

Le cas de la figure 10 correspond ici à un minimum de résistance, en termes de contraintes. On verra plus loin qu'il s'agit aussi d'une enveloppe de chemins de moindre énergie.

- A volume constant, la notion de relation déviatoire est équivalente à celle, utilisée en élastoplasticité, de "potentiel pour les vitesses de déformation ^ (c'est-àdire une fonction des contraintes $F\left(\sigma_{1}, \sigma_{2}, \sigma_{3}\right)$, telles que les vitesses de déformation soient données par $\varepsilon_{1}$ $=\lambda$. $\left.\partial \mathrm{F} / \partial \sigma_{1}\right)$. En effet, la relation déviatoire, ou son inverse $\mathrm{c}=\mathrm{f}^{-\mathrm{I}}$ (b) et la condition de volume constant, peuvent être écrites sous forme d'un système de deux équations indépendantes, linéaires et homogènes par

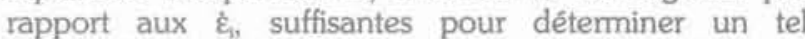
potentiel à une constante additive près. $\mathrm{Ce}$ potentiel, qui n'a ici aucune raison d'être « associé s, s'obtient aisément pour les exemples simples des figures 8 et 10 :

- avec la colinéarité des déviateurs (fig. 8) on trouve un potentiel de Von Mises,

- dans le cas du critère de Coulomb (fig. 10), on obtient un potentiel de Tresca.

Enfin, si la relation déviatoire a déjà pu être mesurée directement en sollicitation tridimensionnelle - au pic, il est vrai, cf. DARVE (1983) - la mesure d'un potentiel des vitesses semble moins évidente.

\subsection{Chemins de moindre énergie}

- On peut se demander si la relation déviatoire est bien une réalité intrinsèque, c'est-à-dire physiquement une sorte de résultat statistique lié aux relations entre efforts et mouvement intergranulaires, ou s'il s'agit seulement d'une observation, qui dépend des conditions de mesure et de sollicitation.

Si la notion de relation déviatoire n'est qu'une observation qui dépend des conditions de mesure, le matériau suit vraisemblablement un chemin de moindre énergie, qui doit être compatible, à l'état critique, avec:

- les conditions imposées par la sollicitation:

- la relation de volume constant;

- l'équation de dissipation.

Analytiquement, la notion de moindre énergie se traduit par un minimum (lié) de la puissance de déformation.

- Considérons la famille de sollicitations tridimensionnelles, très utilisée par les expérimentateurs, où les valeurs de $\sigma_{3}$,

$$
\mathrm{b}=\left(\sigma_{2} \sigma_{3}\right) /\left(\sigma_{1} \sigma_{3}\right) \text {, et } \dot{\varepsilon}_{1}
$$

sont maintenues constantes. 


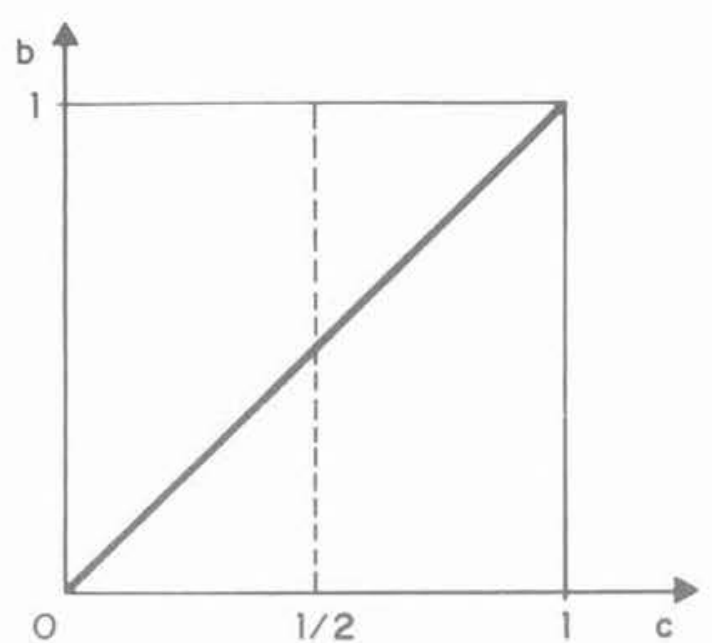

(a) Relation déviatoire

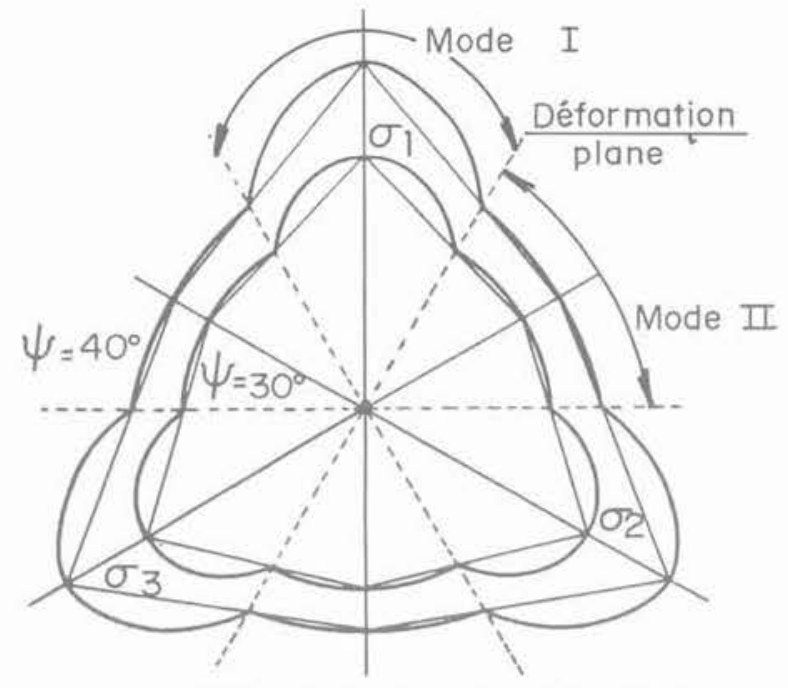

b. Critère de rupture à l'état critique

Fig. 8 - a. Relation déviatoire.
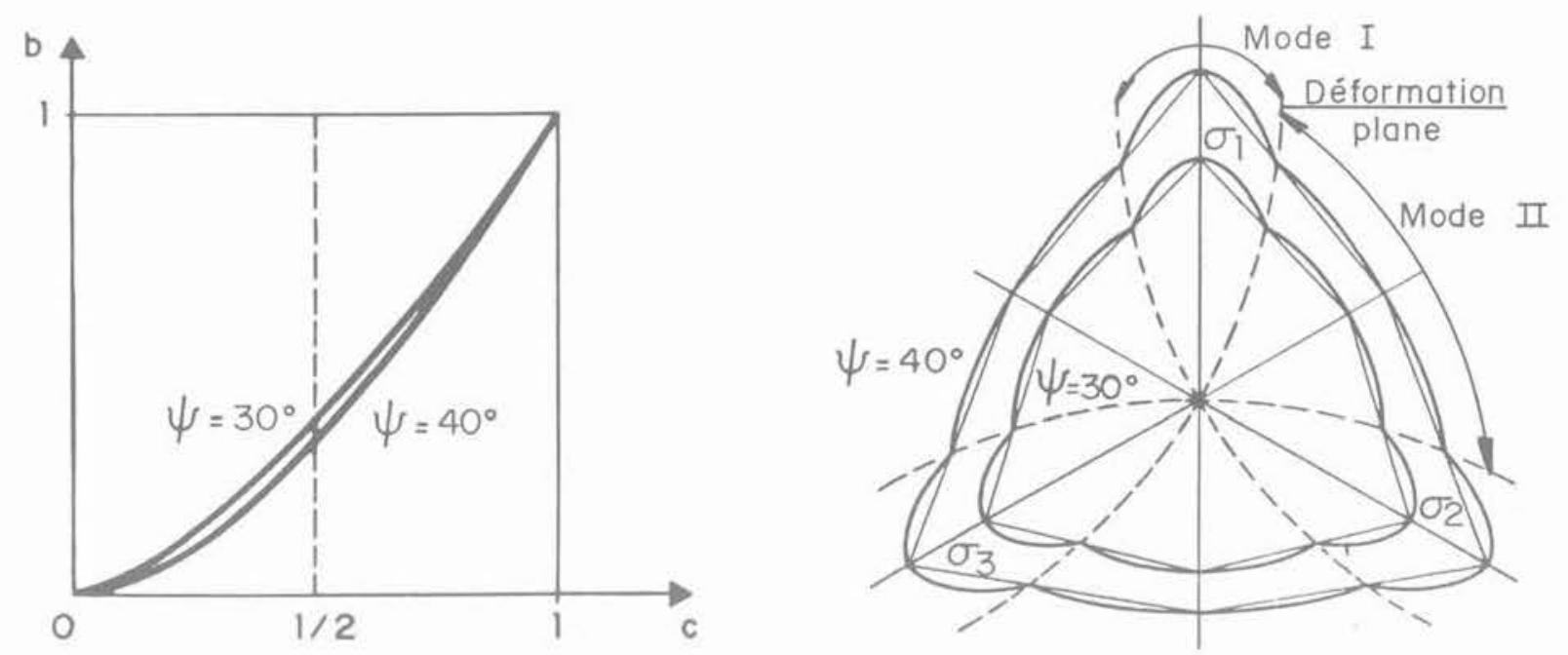

Fig. 9
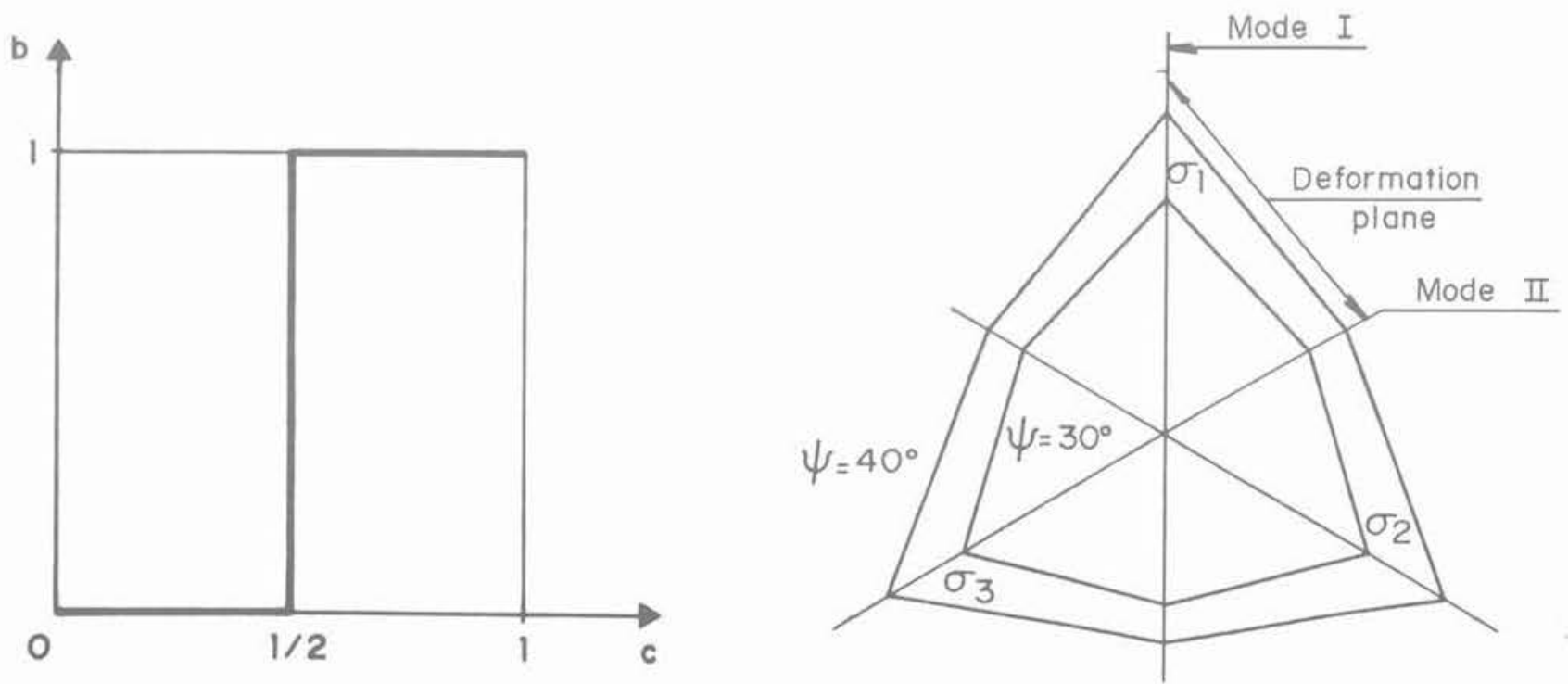

Fig. 10 
On peut tirer la puissance de déformation des équations développées en annexe II, en fonction de $\sigma_{3}, \varepsilon_{1}, \quad b$ et $c$, puis chercher la relation $c(b)$ qui minimise cette puissance de déformation:

- en mode I, on obtient, en notant $\mathrm{S}=\sin \psi$ :

$$
\begin{gathered}
\mathrm{P}=\sum_{1} \sigma_{i} \dot{\varepsilon}_{i}=2 \sigma_{3} \varepsilon_{1}\{\mathrm{c}(2 \mathrm{~b}-1)+(2-\mathrm{b})\} \\
/\{\mathrm{c}[2 \mathrm{~b}(1+\mathrm{S})-(1-\mathrm{S})]-[\mathrm{b}(1+\mathrm{S})-2(1-\mathrm{S})]\}
\end{gathered}
$$

$\mathrm{P}$ prend la même valeur $\mathrm{P}_{0}=2 \sigma_{3} \dot{\varepsilon}_{1} \cdot \mathrm{S} /(1-\mathrm{S})$ en symétrie axiale en contrainte $(b=0)$ ou en déformation plane $c=1 / 2$. Cette valeur $P_{0}$ est bien le minimum de $\mathrm{P}$ sur le domaine du mode I $(0<c<1 / 2)$ et $\mathrm{b}>0$ ) car la dérivée de $\mathrm{P}$ par rapport à $\mathrm{c}$ vaut:

$$
\partial \mathrm{P} / \partial \mathrm{c}=-12 \sigma_{\dot{\xi}} \dot{\xi}_{2}, \mathrm{~b} . \mathrm{S}^{2} / \mathrm{D}^{2}
$$

où $\mathrm{D}$ est le dénominateur dans l'expression précédente de $P$.

Pour $\mathrm{b} \neq 0$, on voit que $\mathrm{aP} / 2 \mathrm{c}<0$.

La fonction $\mathrm{P}(\mathrm{c})$ est donc monotone décroissante et son minimum, sur le domaine du mode I $(0<c<1 / 2)$ est atteint pour la borne supérieure $c=16 / 2$, où $P$ vaut précisément $P_{0}=2 \sigma_{3} \varepsilon_{1} \cdot S /(1-S)$.

Le chemin de moindre énergie en mode l, pour cette famille de sollicitations, est donc le régime de déformation plane $c=1 / 2$; indépendamment de la valeur donnée à b.
- En outre, si l'on impose la symétrie axiale en contraintes $(b=0)$, tous les régimes de déformation intermédiaires entre le triaxial compression et la déformation plane sont également possibles, de manière physiquement stable, car la dérivée de $\mathrm{P}$ par rapport à $\mathrm{c}$ s'annule pour $\mathrm{b}=0$, et $\mathrm{P}$ est alors égal à sa valeur minimale $P_{0}$.

- en mode II :

$$
\mathrm{P}=\sum_{1} \sigma_{1} \varepsilon_{1}=2 \sigma_{3} \varepsilon_{1} \cdot S(1+\mathrm{c}) /(1-\mathrm{S})(2-\mathrm{c})
$$

Le minimum de cette fonction monotone croissante de $\mathrm{c}$ a lieu pour la borne inférieure du mode II $c=1 / 2$; et le maximum a lieu pour la borne supérieure $\mathrm{c}=1$.

Comme en mode I, le chemin de moindre énergie est encore le régime de déformation plane, indépendamment de la valeur de b. A la différence du mode 1, la symétrie axiale en déformation ( $c=1)$ ne peut être un minimum, il s'agit même d'un maximum, physiquement instable car la dérivée de $\mathrm{P}$ par rapport à c ne s'y annule pas.

- Dans une sollicitation monotone à b constant, le matériau évoluerait donc vers un régime limite de déformation plane à volume constant, quel que soit $\mathrm{b}$, sauf peut être pour $\mathrm{b}=0$. Le critère de rupture ainsi obtenu, figure 11 , est donc le critère de Coulomb. (cf. \$ 3.2).

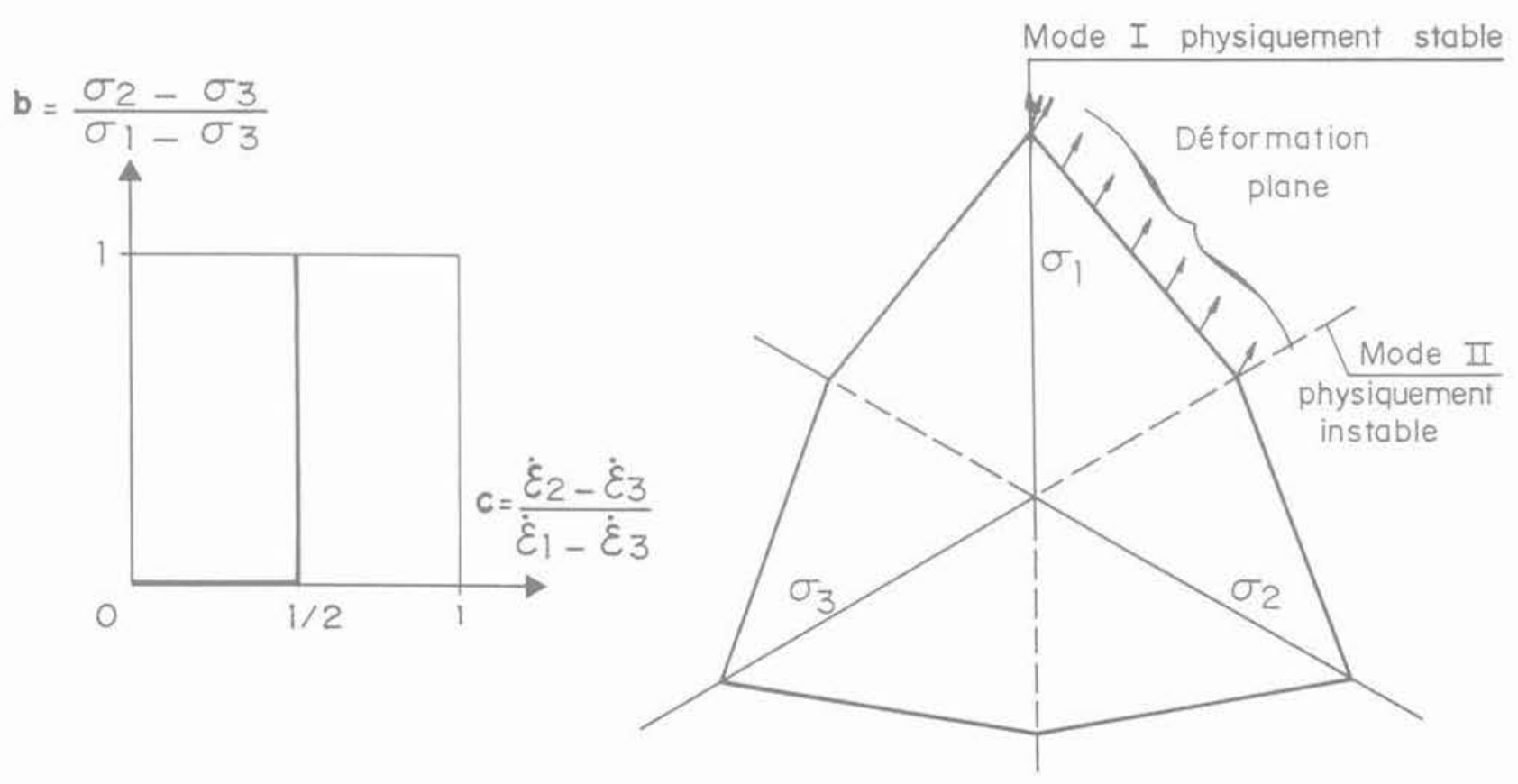

(a) Relation déviatoire

(b) Critère de rupture

Fig. 11. - Enveloppe des chemins de moindre énergie, prévue par l'équation de dissipation, à l'état critique, pour des sollicitations à $\sigma_{3}$ et $b$ constants. 
Certains expérimentateurs sur presse tridimensionnelle ont noté que, dans un essai monotone à $\mathrm{b} \neq 0$ constant, on voit apparaître un mécanisme de déformation plane, localisé, alors que l'emploi de plaques rigides aux frontières de l'éprouvette semblait garantir a priori une certaine homogénéité de déformation (LANIER, 1984-b). Une explication théorique à cette tendance à la déformation plane est suggérée par le développement précédent, sa localisation ou non est une autre question. (DESRUES, LANIER, STUTZ 1984).

L'essai triaxial classique en compression impose la symétrie axiale en contrainte, $\mathrm{b}=0$ et les expérimentateurs savent bien que, malgré les précautions sévères (embases lubrifiées, homogénéisation soignée à la mise en place, etc.) on peut aussi bien voir apparaître comme schéma de déformation, sans raison vraiment apparente:

- une déformation homogène symétrique;

- une déformation homogène avec ovalisation de l'éprouvette:

- la formation d'un genou dans l'éprouvette (que l'on attribue ici à une zone de déformation plane) qui finit par se localiser en un plan de cisaillement.

Le développement théorique précédent souligne cette espèce d'indétermination physique quand $\mathrm{b}=0$ (triaxial compression).

Enfin, il est notoire que si les essais triaxiaux en compression peuvent être stables, ce qui permet de les poursuivre jusqu'à de larges déformations, les essais triaxiaux en extension sont toujours instables.

L'analyse des chemins de moindre énergie suggère des raisons théoriques à cet état de fait.

\section{DISCUSSION}

- ROWE et HORNE, qui décrivaient leurs résultats en symétrie axiale en termes de «incremental input energy \& et de a incremental output energy s avaient bien sûr clairement identifié la nature physique du rapport $\sigma_{1} \varepsilon_{1} / 2 \sigma_{3} \dot{\varepsilon}_{3}$ (tableau I). ROWE note de plus en 1969 à propos de la déformation plane: * la relation [contraintes-dilatance]... constitue simplement une généralisation de l'expression de COULOMB-RANKINE pour inclure des taux de dilatance différents de l'unité ».

De vives discussions s'étaient alors développées autour de ces notions en particulier à cause d'un certain manque d'objectivité (cf. DE JOSSELIN DE JONG (1976)).

- Avec les définitions données au $\$ 1-2$, les termes de "puissance reçue * et de * puissance retransmise * trouvent une expression objective, que l'on peut étendre aux situations tridimensionnelles.

Sur les chemins variés, le rapport expérimental de ces puissances paraît constant, indépendant de l'état initial, de la pression de confinement, et de la contrainte intermédiaire. Pour l'auteur, ceci suffit à justifier ce que l'on propose comme un principe, dont on ne connaît pas pour l'instant la réduction à des causes physiques plus élémentaires.

- On peut penser que ce schéma décrit approximativement un mécanisme micro-structural de déformation correspondant au glissement relatif de grains quasirigides en contact frictionnel.

D'autres mécanismes peuvent devenir prépondérants sur certains chemins de sollicitation (cf \$2.2), ils ne sont pas représentés ici.

Ceci peut se produire lorsque les grains en contact ne peuvent pas être considérés comme quasi-rigides. En particulier, pour une sollicitation de type oedométrique, qui impose un même signe - au sens large à toutes les vitesses de déformation principale, l'équation de dissipation indique théoriquement un blocage de la déformation pour $\psi \neq \Pi / 2$ (la seule solution possible dans (3) est $\dot{\varepsilon}=0$ ).

Cet état de fait donne quelques indications sur le domaine de validité de l'équation de dissipation: elle schématiserait approximativement ce qui se passe sur des chemins de sollicitation drainés, essentiellement déviatoires, à dominante monotone, où la pression moyenne ne subit que des variations modérées et lentes. Sur de tels chemins, l'hypothèse de coaxialité entre les tenseurs $\underline{\sigma}$ et $\underline{\dot{\varepsilon}}$ semble raisonnable.

- Ce schéma permet une analyse de la notion de critère de rupture à l'état critique, qui * colle » à bien des faits observés, il expliquerait pourquoi le critère de COULOMB demeure le critère expérimental de référence (cf. ARTHUR et al. 1977), tout particulièrement en comportement à volume constant de l'état critique (VARDOULAKIS et GOLDSCHEIDER, 1981).

Le critère de rupture «au pic * n'est pas accessible par la seule équation de dissipation combinée avec l'une des notions de relation déviatoire ou de moindre énergie.

En effet, la notion de « pic » désigne un état extrémal au cours d'une évolution du matériau, dénommée usuellement « écrouissage ». L'approche des conditions " au pic "nécessite donc une schématisation de cette évolution.

Les critères de rupture à l'état critique obtenus par l'équation de dissipation et la notion de relation déviatoire, coincident toujours avec celui de COU$\mathrm{LOMB}$ en symétrie axiale et en déformation plane.

En dehors de ces chemins simples l'approche en moindre énergie indique le même critère de COULOMB.

Une validation expérimentale directe de l'approche en moindre énergie passerait donc par une expérimentation tridimensionnelle à l'état critique, ce qui paraît difficile compte tenu des effets de localisation de la déformation sous forme de bandes de cisaillement. 
Pour lauteur, ces effets ne remettraient pas en cause la notion même d'état critique, mais simplement en gênent l'observation dans un volume homogène suffisant pour la mesure.

\section{ANNEXE 1}

\section{Cas de signe possibles dans l'équation de dissipation}

à volume constant, respectant les conditions sur la relation déviatoire:

- Les contraintes principales effectives, qui sont des compressions, sont positives ;

- Les directions principales étant indicées suivant l'ordre des contraintes principales, les permutations possibles sur les signes des vitesses de déformation principales conduisent à distinguer $2^{3}=8$ cas (Tableau A-1).

Tableau A-1. - Les 8 permutations possibles sur les signes des $\hat{\varepsilon}_{i}$

\begin{tabular}{|c|c|c|c|c|c|c|c|c|}
\hline & 1 & 2 & 3 & 4 & 5 & 6 & 7 & 8 \\
\hline$\varepsilon_{1}$ & + & + & + & + & - & - & - & - \\
\hline$\varepsilon_{2}$ & - & + & - & + & - & + & - & + \\
\hline$\varepsilon_{3}$ & - & - & + & + & + & + & - & - \\
\hline
\end{tabular}

- A volume constant, les vitesses de déformation principales ne peuvent avoir toutes le même signe, ce qui exclut les permutations $\underline{4}$ et $\underline{7}$.

- On a admis que $0<\mathrm{c}<1$, ce qui implique que $\dot{\varepsilon}_{2}$ est vitesse de déformation intermédiaire car:

$$
\begin{cases}\dot{\varepsilon}_{2}=c \dot{\varepsilon}_{1}+(1-c) \dot{\varepsilon}_{3} & \left.\dot{\varepsilon}_{1}>\dot{\varepsilon}_{2}>\dot{\varepsilon}_{3} \alpha\right) \\ \frac{\text { et }}{0}<c<1 & \left.\frac{\text { ou }}{\dot{\varepsilon}_{1}}<\dot{\varepsilon}_{2}<\dot{\varepsilon}_{3} \beta\right)\end{cases}
$$

Ceci exclut les permutations 3 et $\underline{8}$ dans le tableau A-1.

- Par convention $\sigma_{1}>\sigma_{2}>\sigma_{3}$; par ailleurs les $\varepsilon_{1}$ vérifient la condition de volume constant. On vérifie alors sans peine que les permutations correspondant aux inégalités $\beta$ ), (5 et 6 dans le tableau A-1) rendent négative la puissance des efforts intérieurs

$\mathrm{P}=\sum \sigma \dot{\varepsilon}_{1}$, ce qui est exclu par l'équation de dissipation.

Les seuls cas possibles sont donc les permutations 1 et 2 dans le tableau A-1.

\section{ANNEXE II}

\section{A.2.1. Résolution du système (5)}

- Il est utile d'exprimer les contraintes en fonction de $\sigma_{1}$, de la pression moyenne $\mathrm{p}=\left(\sigma_{1}+\sigma_{2}+\sigma_{3}\right) / 3$, et du paramètre $b$ :

$$
\left\{\begin{array}{l}
\sigma_{2} / p=\left[3(1-b)+(2 b-1) \sigma_{1} / p\right] /(2-b) \\
\sigma_{3} / p=\left[3-(1+b) \sigma_{1} / p\right] /(2-b)
\end{array}\right.
$$

De même, pour les vitesses de déformation, en fonction de $\hat{\varepsilon}_{1}$ et du paramètre c, compte tenu de la condition de volume constant:

$$
\left\{\begin{array}{l}
\dot{\varepsilon}_{2}=-\dot{\varepsilon}_{1}(1-2 \mathrm{c}) /(2-c) \\
\dot{\varepsilon}_{3}=-\dot{\varepsilon}_{1}(1+c) /(2-c)
\end{array}\right.
$$

Compte tenu des signes des $\dot{\varepsilon}_{\text {, }}$, l'équation de dissipation s'écrit :

- Mode I $(1-\sin \psi) \sigma_{1} \dot{\varepsilon}_{1}+(1+\sin \psi)$

$\left(\sigma_{2} \dot{\varepsilon}_{2}+\sigma_{3} \dot{\varepsilon}_{3}\right)=0$

- Mode II $(1-\sin \psi)\left(\sigma_{1} \dot{\varepsilon}_{1}+\sigma_{2} \dot{\varepsilon}_{2}\right)$

$+(1+\sin \psi) \sigma_{3} \dot{\varepsilon}_{3}=0$

- A partir des systèrnes (6), (7) et des équations (8), on obtient:

- en mode 1

$$
\begin{aligned}
& \sigma_{1} / p=3(1+\sin \psi)(2-c-b+2 b c) /[6 b c \sin \psi \\
& +(3-\sin \psi)(2-c-b+2 b c)]
\end{aligned}
$$

- en mode II

$$
\begin{aligned}
& \sigma_{1} / P=3[2-b-c+2 b c+\sin \psi \cdot(b+3 c-2 b c)] \\
& /[(3+\sin \psi)(2-b-c+2 b c)-6 \sin \psi . \\
& (1-b)(1-c)]
\end{aligned}
$$

- Etant donné c, la relation déviatoire donne la valeur de $b$, d'où $\sigma_{1} / p$ par les équations (9), et $\sigma_{2} / p$ ainsi que $\sigma_{3} / \mathrm{p}$ par le système (6).

\section{A.2.2. Position relative par rapport à la pyramide de Coulomb}

- Pour une relation déviatoire donnée $b=f(c)$, on obtient un critère de rupture $\mathrm{Cf}$ par la méthode précédente. En un point courant de $\mathrm{Cf}$, on peut toujours définir un angle de frottement interne apparent par:

$$
\sin \phi=\left(\sigma_{1}-\sigma_{3}\right) /\left(\sigma_{1}+\sigma_{3}\right)
$$

On sait que $\phi=\psi$ en déformation plane ou en symétrie axiale en contraintes.

Notons Co la pyramide de Coulomb obtenue avec un frottement interne égal à $\psi$. Si $\sin \phi>\sin \psi$ le point de $\mathrm{Cf}$ considéré est à l'extérieur de la pyramide de Coulomb $\mathrm{Co}$, si $\sin \phi>\sin \psi$ le point considéré sur $\mathrm{Cf}$ est à l'intérieur de Co. 
- A partir de l'expression des contraintes donnée en A-2.1 et de la définition de $\sin \phi$, on trouve:

- en mode $1(0<c<1 / 2)$

$$
\begin{aligned}
& \sin \phi / \sin \psi-1=(1+\sin \psi) \cdot b \cdot(1-2 c) \\
& /\{1+c+(1-2 c)[1-b(1-\sin \psi)]\}
\end{aligned}
$$

- en mode II $(1 / 2<\mathrm{c}<1)$

$$
\sin \phi / \sin \psi-1=2(1-\sin \psi)(1-b)(2 c-1)
$$$$
/\{3+(2 \mathrm{c}-1)[2 \mathrm{~b}-1+2(1-\mathrm{b}) \sin \psi]\}
$$

Compte tenu de la définition des modes et du fait que $0<b<1$, on vérifie que les dénominateurs et numérateurs des expressions (10) restent positifs, seuls les numérateurs pouvant s'annuler.

Les $\operatorname{cas} \sin \phi=\sin \psi$ ne se produisent, en mode 1 , que si $b=0$ (symétrie axiale en contraintes) ou si $c=1 / 2$ (déformation plane) et en mode ll que si $\mathrm{b}=1$ (symétrie axiale en contrainte) ou si $\mathrm{c}=1 / 2$.

Dans tous les autres cas, les expressions (10) montrent que $\sin \phi>\sin \psi$, donc:

Quelle que soit la relation déviatoire $b=f(c)$, le critère de rupture obtenu $(C f)$ est toujours à l'extérieur de la pyramide de Coulomb (Co) sauf en déformation plane, et en symétrie axiale en contraintes, auxquels cas il y a coïncidence.

\section{NOTATIONS}

b Paramètre de Habib $\mathrm{b}=\left(\sigma_{2}-\sigma_{3}\right) /\left(\sigma_{1}\right.$ $\left.-\sigma_{3}\right)$ Homologue du paramètre $b$

c Homologue du paramètre

$\mathrm{P}_{+}, \mathrm{P}_{-}, \mathrm{P}, \mathrm{Po}$ Puissances développées dans la déformation, rapportées à l'unité de volume

s' Moyenne des contraintes principales extrêmes

t' Cisaillement maximal

$\mathrm{X}(\mathrm{T}), \mathrm{Y}(\mathrm{T})$ Energies développées dans la déformation, rapportées à l'unité de volume

$\sigma \quad$ Contraintes (effectives)

$\varepsilon \quad$ Vitesse de déformation

V Vitesse de distorsion maximale

$\checkmark \quad$ Vitesse de déformation de volume

$\phi_{\mathrm{cv}} \quad$ Frottement interne à volume constant

à l'état critique

$\phi_{\text {pic }} \quad$ Frottement interne au pic

$\phi_{\mu} \quad$ Angle de friction grain sur grain

$\phi_{1} \quad$ Angle de friction mesuré par la théorie de Rowe

$\psi \quad$ Angle d'écoulement

\section{BIBLIOGRAPHIE}

ARTHUR J.R.F., DUNSTAN T., AL-ANI Q.A.J.L., ASSADI A. (1977). - *Plastic deformation and failure in granular media * Géotechnique 27, $\mathrm{n}^{\circ} 1,53-74$.

BARDEN L., ISMAIL H., TONG P. (1969). - «Plane strain deformation of granular material at low and high pressures ». Géotechnique 19, n4, 441452.
BARDEN L., KHAYATT A.J. (1966). - - Incremental strain rate ratios and strength of sand in the triaxial test ». Géotechnique 16, $\mathrm{n}^{\circ} 4,338-357$.

CAMBOU B. (1985). - "Les micromécanismes de déformation plastique dans un sol granulaire ». Revue française de Géotechnique $n^{\circ} 31$.

CHARLES J.A. WATTS K.S. (1980). - "The influence of confining pressure on the shear strength of compacted rockfill ». Géotechnique 30 , $n^{\circ} 4,143-167$.

CORNFORTH D.H. (1964). - « Some experiments on the influence of strain conditions on the strength of sand». Géotechnique 14, $\mathrm{n}^{\circ} 12,143-167$.

DARVE F. (1983). - - Lois de comportement des sols sous sollicitations tridimensionnelles et cycliques *. Réunion-débat du 23.03.1983, Annales de III.T.B.T.P., n 432 , février-mars 1985.

DE JOSSELIN DE JONG (1976). _ « Rowe's stress dilatancy relation based on friction 》. Géotechnique $26, \mathrm{n}^{\circ} 3,527-534$; discussion by $\mathrm{G}$. GUDEHUS (1977) 27, $n^{\circ} 3,439$

DESRUES J., LANIER J., STUTZ P. - - Localization of deformation in tests on sand samples.. Engineering Fracture Mechanics, 1984.

FROSSARD E. (1983), - "Une équation d'écoulement simple pour les matériaux granulaires ”. Géotechnique $33, n^{\circ} 1,21-29$.

HORNE M.R. (1965). - « The behaviour of an assembly of rotund, rigid, cohesionless particles ». Part I and II. Proc. R. Soc. series A-286, 62-97.

HORNE M.R. (1969). - «The behaviour of an assembly of rotund, rigid, cohesionless particles ». Part III. Proc. R. Soc. series A-310, 21-34.

HUGUES J.M.O., WROTH C.P., WINDLE D. (1977). - « Pressuremeter tests in sands ». Géotechnique 27, $\mathrm{n}^{\circ} 4,455-477$.

LAMBE T.W., WHITMAN R.V. (1969). - «Soils Mechanics ». New York, Wiley.

LANIER J. (1984 b). - «Développements récents des essais mécaniques en laboratoire ». Ecole d'hiver de rhéologie des géomatériaux I.M.G., C.N.R.S.

RAMAMURTHY T. TOKHI V.K. (1961). - «Relation of triaxial and plane strain strength *. Proc. 10th Int. Conf. on Soil Mech. and Found. Eng. Stockholm.

ROWE P.W. (1962). - « The stress dilatancy relation for static equilibrium of an assembly of particles in contact ». Proc. R. Soc. series A-269, 500527.

ROWE P.W. (1969). - - The relation between the shear strength of sands in triaxial compression, plane strain and direct shear ». Géotechnique 19, $\mathrm{n}^{\circ} 1,75-86$.

SCHOFIELD A.N., WROTH C.P. (1968). - «Critical state soil Mechanics ». Mc Graw-Hill.

TAYLOR D.W. (1948). - *Fundamentals of soil mechanics ». New York, Wiley.

VARDOULAKIS I., GOLDSCHEIDER M. (1981). "Biaxial apparatus for testing shear bands in soils „. Proc. 10th Int. Conf. on Soil Mech. and Found. Eng. Stockholm. 